\title{
Optoelectronics for electrical and computer engineering students
}

\section{Soo-Jin Chua, Mansoor Jalil}

Soo-Jin Chua, Mansoor Jalil, "Optoelectronics for electrical and computer engineering students," Proc. SPIE 4588, Seventh International Conference on Education and Training in Optics and Photonics, (28 May 2002); doi: 10.1117/12.468699

SPIE Event: Education and Training in Optics and Photonics 2001, 2001, Singapore, Singapore 


\title{
Optoelectronics for electrical and computer engineering students
}

\author{
S. J. Chua and M. B. A. Jalil \\ Department of Electrical and Computer Engineering, \\ National University of Singapore, Singapore 117576.
}

\begin{abstract}
We describe the contents of an advanced undergraduate course on Optoelectronics at the Department of Electrical and Computer Engineering, National University of Singapore. The emphasis has changed over the years to keep abreast of the development in the field but the broad features remain the same. A multidisciplinary approach is taken, incorporating physics, materials science and engineering concepts to explain the operation of optoelectronic components, and their application in display, communications and consumer electronics. The course comprises of 36 hours of lectures and two experiments, and covers basic radiometry and photometry, photoemitters (LEDs and lasers), photodetectors, and liquid crystal displays. The main aim of the course is to equip the student with the requisite theoretical and practical knowledge for participation in the photonics industry and for postgraduate research for students who are so inclined.
\end{abstract}

Keywords: optoelectronics course, LEDs, lasers, photodetectors, liquid crystal displays

\section{$1 \quad$ Introduction}

An advanced undergraduate course on Optoelectronics has been taught to final-year students at the Department of Electrical and Computer Engineering at the National University of Singapore for the past 13 years. The contents of the course are constantly updated to keep abreast of the latest technological and product developments in the field. Topics are included in the course based on their relevance to the growing optoelectronics-related industry in Singapore, so that students will have the requisite knowledge and expertise for employment in the industry. For instance, with the surge in importance in optical communications and use of optoelectronics for consumer products, many of the multinational companies such as Agilent, Philips, Sony, Fujitsu, Avimo and others are in need of manpower with the knowledge on optical components for these applications. Also a large investment in liquid crystal display plant by Toshiba, proposed for completion in 2003, has led to a greater emphasis on liquid crystal technology in the course syllabus. Despite these changes, the basic structure of the course has generally been retained. The main objective of the course is to equip the student with the basic physics concepts in light production and emission, mainly in semiconductors, and their applications in display components and devices and optical communications. Two experiments on optical heterodyning and output characteristics of semiconductor lasers and LEDs, respectively, are designed to provide a practical hands-on experience to complement the lectures. The course is divided into two main sections: i) Basic photometry and photoemitters (LEDs and lasers) and ii) photodetectors, electro-optic modulation and liquid crystals.

2

Photometry and radiometry

Radiometry and photometry is the science of measurement of radiation and forms an essential part in quantifying the performance of optical devices. Both types of units are introduced since there are applications where only the energy aspect of radiation is important and hence, radiometric units are used, whereas photometric units are used when the 
visual aspect is the main concern. A sound understanding of the use of units and techniques for calculating radiative transfer are necessary for solving practical problems involving optical fluxes. The units are introduced and defined as follows:

\section{Radiometric Photometric}

$\begin{array}{llll}\text { Incidance } & E=\frac{d \phi}{d A} & \text { Watt } / \mathrm{m}^{2} & \text { Lumen } / \mathrm{m}^{2} \\ \text { Exitance } & M=\frac{d \phi}{d A} & \text { Watt } / \mathrm{m}^{2} & \text { Lumen } / \mathrm{m}^{2} \\ \text { Intensity, } & I=\frac{d \phi}{d \omega} & \mathrm{Watt} / \mathrm{sr} & \text { Lumen } / \mathrm{sr} \text { (Candela) } \\ & & & \\ \text { Sterance, } & L=\frac{d I}{d A} & \mathrm{Watt} / \mathrm{m}^{2} . \mathrm{sr} & \text { Watt } / \mathrm{m}^{2} . \mathrm{sr}\end{array}$

Fig. 1 Generic Terms and Symbols and their Geometrical Relationships

Corresponding to the generic terms are the following radiometric and photometric terms: Incidance - Irradiance and Illuminance; Sterance - Radiance and Luminance and Exitance - Radiant Exitance and Luminous Exitance. A good understanding of the relationship of the above-mentioned units is important for solving practical problems. The sensation of brightness perceived by the eye, evoked by radiant energy at various wavelength is measured in photometric units and the relationship to the energy content is given by the Luminosity function or the luminosity curve or the "CIE" curve. The peak in the luminosity curve occurs at a wavelength of $0.555 \mu \mathrm{m}$ and at that wavelength, 1 Watt of radiant flux corresponds to a luminous flux of 680 lumens. Through this relationship, luminous efficiency can be obtained for any light emitting device. Lamberts law is presented so as to introduce the concept of a perfectly diffused surface. With the introduction of the units, and their relationships and the nature of surfaces, typical problems are solved, three of which are illustrated below.

1) An LED emits light of an intensity with a known angular dependence. It is required to calculate the illuminance at a surface some distance away and with a defined angular orientation.

2) A laser beam with a given intensity irradiates an incline surface at a distance away. The surface is assumed to be perfectly diffused. It is required to find the irradiance, say at a detector surface located near the laser source as a result of the laser light scattered from the surface.

3) A monochrome display gives out certain brightness at a given wavelength. The operating voltage and input current are given. It is required to calculate the quantum efficiency and the power conversion efficiency.

Many other practical problems related to optical flux transfers are discussed and solved. Approximations are introduced where applicable to simplify the calculations. 


\subsection{Photoemitters}

\section{Material properties}

Photoemitters are semiconductor lasers and LEDs and they come in different structures and wavelength emissions. The questions to answer are therefore why the variety and what material properties control their operation. Semiconductor lasers and LEDs are fabricated from compound semiconductors and are described most importantly by their energy bandgaps and lattice constants. To obtain a general perspective of the variety, the important compound semiconductors are introduced to the students as plotted in Fig. 2. The lines joining the binary compounds give the lattice constant/bandgap variation of the ternary compounds while areas bounded by the boundary curves give the compositions of the quaternary.

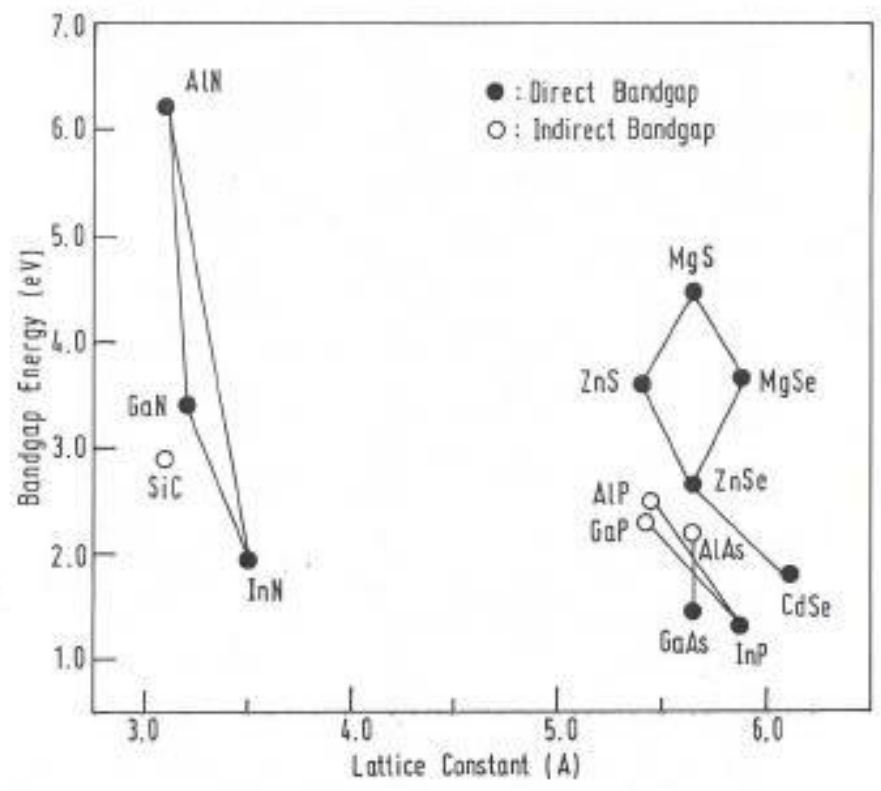

Fig. 2 Energy bandgap versus lattice constants of important binary, ternary and quaternary compound semiconductors.

The concept of lattice matching, pseudomorphic layers and the effects of strain on the heavy and light hole sub-bands are introduced. In particular under biaxial tensile strain, the light hole is lower in energy than the heavy hole and the bandgap shrinks. Calculations are carried out using Vegard's law for relating lattice constants to the ternary or quaternary compositions. These provide the students with an appreciation of how bandgap can be adjusted while maintaining lattice matching to the substrate. Many optoelectronic devices also make use of the band offsets at the conduction and valence bands as potential barrier for the confinement of electrons and holes, respectively. As an illustration, the electron injection efficiency was derived for the $\mathrm{p}-\mathrm{N}$ homojuntion and the $\mathrm{p}-\mathrm{N}$ heterojunction, which clearly showed that holes can be effectively blocked from crossing the junction with the hole current reduced by the Kroemer factor. As a result of the relative band alignment, types I and II are defined as illustrated in Fig.3. Band bending occurs as a result of carrier movement to maintain energy equilibrium and they play a part in type II optical transitions.

Another important application of bandgap discontinuity and band offsets is in the double heterostructure and quantum well lasers and in high electron mobility transistors. The reason for using the multilayers becomes apparent once the band diagrams are illustrated to indicate the confinement of carriers to certain layers. The concept of heterostructures formed by layers of different bandgap materials and band offsets allows for the appreciation of bandgap engineering. Novel structures such as the staircase avalanche photodiodes and graded gap heterostructure bipolar transistor can now be made using the interesting property of a built-in field. Further examples are discussed in the section on 
photodetectors. With an appreciation of the material composition and the related bandgaps, the students are in a better position to understand the reason for the various layers used in the structure of semiconductor lasers and LEDs.

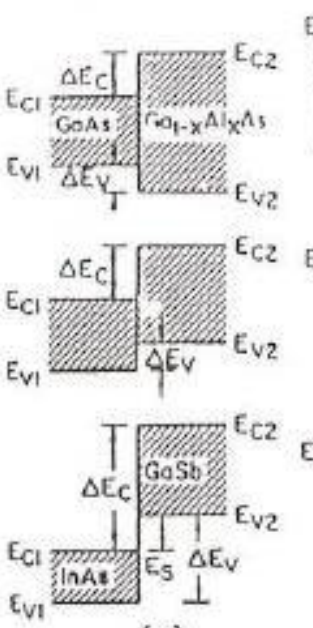

(a)
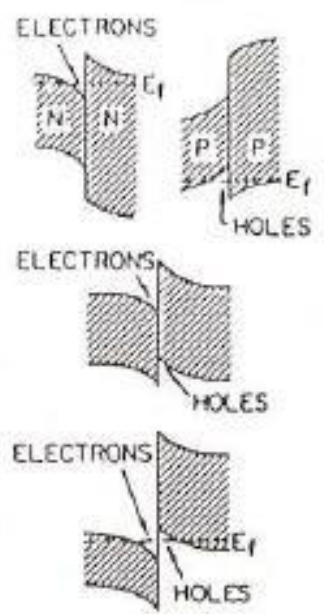

(b)
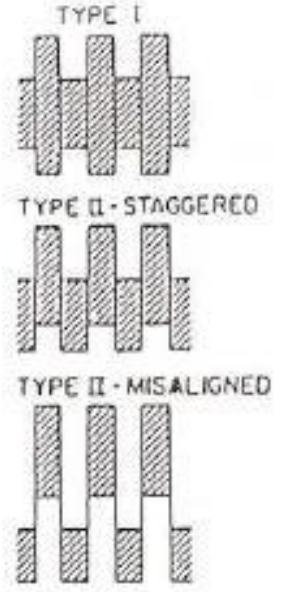

(c)

Fig.3 Type I and II band alignments. (a) physically separated, (b) in equilibrium resulting in band bending and (c) superlattice band structure.

\subsection{Semiconductor lasers}

Semiconductor lasers are used for optical communications, data storage, barcode scanning, laser ranging and many others. However the most stringent of the applications is in optical communications requiring single longitudinal mode operation, linear light current charceristics, low threshold, low temperature drift and high modulation rate. Thus the focus is in understanding the factors that determine these parameters. The rate equations ${ }^{1}$ are introduced to provide the mathematical framework for the analysis of the light-current characteristics. In particular the terms such as spontaneous lifetime, photon lifetime, gain coefficient, optical confinement factor, optical feedback factor and transparency carrier concentration are introduced. The threshold condition is derived using the idea that the round trip gain through the cavity is equal to 1 . Thus with these equations, the student is able to determine from the experimental light-current characteristics, the differential quantum efficiency, the characteristic temperature $\mathrm{T}_{0}$, and from the spectrum, the cavity length and from the turn-on time delay of the optical pulse with respect to the current pulse, the carrier lifetime. From the distribution of gain with carrier injection and the density of states distribution with energy, the effect of temperature in modifying the Fermi distribution function, prediction of the effects of current density and temperature on the light output spectrum can be deduced. The various laser structures are introduced, such as the buried heterostructure, channeled substrate planar, ridge waveguide and others, to give an indication of ingenuity of researchers in avoiding patent issues. The students after going through this section have a good idea of analysing the laser structures and what the various layers are for. Such knowledge is important for analyzing any new devices that may be invented in future.

\subsection{Light emitting diodes - LEDs}

Light emitting diodes make use of the spontaneous emission arising from the recombination of electrons and holes across a direct energy bandgap. The wavelength of the emission is therefore determined by the energy bandgap of the active materials. From the materials presented in Fig. 2, the students become aware of the need of different materials to span the emission wavelengths from the visible to the infra-red. The group III-nitrides is an important family for the blue to green light emitting LEDs. As for the yellow and red, there is the quaternary InGaAlP and for the infrared, the AlGaAs and GaAs:Si materials. One notable example where an indirect bandgap material, viz GaP, is used for green 
light emission, is highlighted. Here the principle of isoelectronic traps, where the presence of vastly different electronegativities between phosphorus and nitrogen atoms, used for creating excitons, is explained. By increasing the concentration of nitrogen dopants, due to their potential interactions, the isoelectronic traps sink deeper into the bandgap and the emission wavelength can be shifted to the yellow. Heterostructure LEDs made of InGaAsP are also introduced. Again the various layers making the LEDs are explained with each layer having a specific function.

One of the differences of LEDs compared to semicondcuctor lasers is the broader spectrum. A simple calculation, whereby the energy spread of a $1 / 2 \mathrm{kT}$ is taken between the electrons and holes in the conduction and valence bands respectively, can account for the observed $20 \mathrm{~nm}$ spectral width. An equation relating to the power output to current density is derived to show its dependence on carrier lifetime. In fact the whole philosophy in imparting the knowledge is to give the students sufficient physics and concepts to enable them to figure out the results obtained and to be able to make educated guesses of what is going on. An experiment to measure the light-current characteristics of lasers and LEDs and their polarization properties, accompanies the course to reinforce what they have learnt in theory. Another experiment on optical heterodyning is designed for students to handle optical measurements, which often requires precise alignment. In this experiment, an acousto-optic modulator modulates a $\mathrm{HeNe}$ laser beam, with the frequency shifted by $80 \mathrm{MHz}$. The signal is recovered by mode beating with the original beam and displayed on an oscilloscope.

\section{$3 \quad$ Photodetectors}

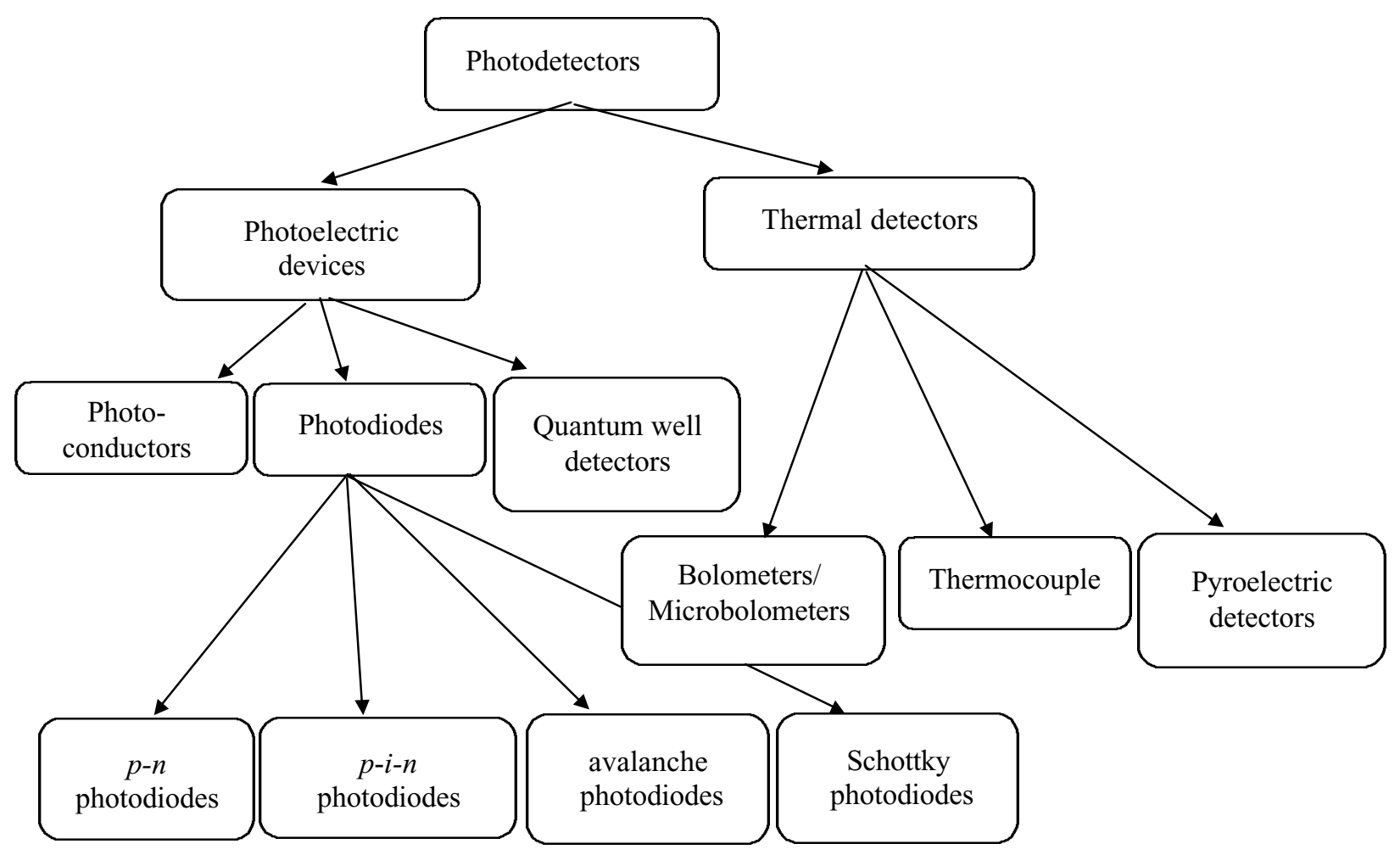

Figure 4. Hierarchy of detectors covered in the course.

The course discusses the most common types of photodetectors, namely: (i) photo-conductors, (ii) photodiodes, (iii) quantum well detectors and (iv) thermal detectors. In our description, we place emphasis on their principal application today, i.e. to transform optical signals, transmitted via optical fibres, into electrical signals. 
Since the course focuses mainly on semiconductor-based photodetectors, we begin by introducing the conduction and valence band-diagrams to explain the photodetection process. This is an elaboration of the basic semiconductor theory, which students would have studied in previous years. The photoexcitation process in direct and indirect band-gap materials is described in some detail, in order to explain the fundamental difference in their optical properties.

For the simplest photodetector, i.e. the photoconductor, the band-diagram is used to depict the gain process, which is the main advantage of photoconductors. Impurity states are then included in the band-diagram to account for the ability of doped photoconductors to detect long wavelength (infra-red) radiation. Before proceeding to more complex devices, various figures of merits pertaining to photodetectors are discussed, such as responsivity, quantum efficiency, dark current, noise, frequency response and bandwidth.

We then proceed to the $p-n$ photodiode and its many variations and refinements, e.g. the $p-i-n$ photodiode, avalanche photodiode (APD) and the Schottky photodiode. We again emphasized on the respective band-diagrams of these detectors so as (i) to explain the underlying physics of device operation in an accessible (pictorial) form and (ii) to show how the band-structure has been deliberately designed in the different types of photodiodes to achieve certain desired properties. These aims are illustrated for two particular APD designs [Figs. 5(a) and (b)]. (i) In a reach-through APD (RAPD), which has a $p-\pi-p-n^{+}$structure, the wide $\pi$ region ensures a high probability of absorption, thus improving its quantum efficiency. RAPD also boasts greater stability because the voltage drop is shared between the $\pi$ and $p-n^{+}$ regions, and the fact that only one type of carrier participates in the avalanche process. (ii) In separate absorption and multiplication (SAM) APDs, a heterostructure is specifically designed, so that absorption occurs in the narrow gap material (e.g. InGaAs), while the avalanche process proceeds at the $p-n^{+}$junction of the wide-gap material (e.g. InP). This eliminates the problem of dark current, which is especially serious in IR detectors $(\lambda \sim 1.3 \mu \mathrm{m})$, used in fibre-optics communications.

The concept of "band-gap engineering", i.e. the interfacing of band diagrams of separate semiconductor materials to produce some desired heterojunction behaviour, is developed further in the discussion on novel quantum well infra-red photodetectors (QWIP). Examples of band-gap engineering include (i) the formation of quantum well by wide-narrowwide bandgap sandwich structure, and (ii) multiple well structure "tilted" by an applied field. The physics of the operation of these devices is explained using introductory quantum mechanical concepts, such as discrete energy levels due to confinement, inter-level transition probability and inter-well tunneling. The energy difference between the discrete levels within the well is typically small, so that QWIPs are well suited for detection of far infrared radiation. Additionally, these levels can be controlled by modifying the well width and depth, so that QWIPs can be designed to give maximum sensitivity at $\lambda=8-12 \mu \mathrm{m}$, which makes them suitable for night-vision applications. A brief mention is made of the advanced techniques (e.g. MBE and MOCVD) used in the precise fabrication of ultra-thin layers within the heterostructures.

A short section on thermal detectors is included, which covers the basic thermodynamic theory involved, and an overview of the three main types, i.e. thermopiles, pyroelectric detectors and bolometers. Of the three, a greater attention is given to bolometers, due to recent advances in microbolometer technology. A typical microbolometer consists of an ultra-thin floating $\mathrm{SiO}_{2}$ membrane suspended above an air recess. We also incorporate nanofabrication techniques, such as anisotropic etching to form the recess, which were developed by researchers at the Centre of Optoelectronics, NUS. ${ }^{2}$ The application of uncooled focal plane array of microbolometers is to function as IR detectors and night vision cameras. 


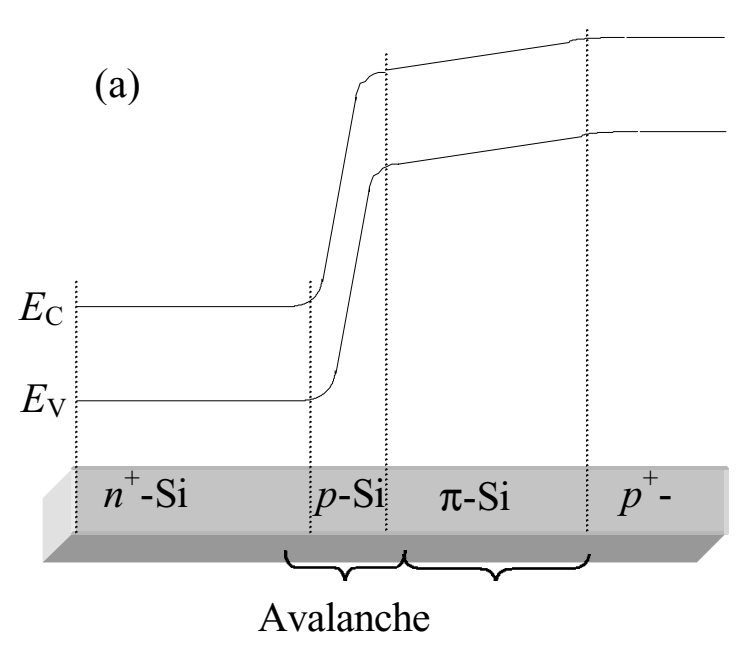

Fig. 5 Band-diagrams of specialised avalanche photodiodes: (a) reach-through APD, (b) separate absorption and multiplication APD.

\section{$4 \quad$ Optical modulation}

So far we have discussed the optical sources and detectors at the terminal ends of a fibre-optics communication system. For completeness, a section on electro-optic modulation is included to cover another major aspect of optical communications, i.e. the conversion of electrical to optical signals, which are then transmitted via the optical fibres. For a ray of light to carry information, it has to undergo some form of modulation of its properties. Basically, this is achieved by passing light through a medium whose refractive index can be modulated by electrical means. Such a medium is said to exhibit either the Pockels or Kerr effect, depending on whether the refractive index $n$ is linearly or quadratically dependent on the electric field.

This modulation in $n$ has to be translated into a modulation of a measurable quantity, e.g. intensity, before it can be utilized. Two types of intensity modulators are described. The first consists of an anisotropic Pockels cell, sandwiched between two crossed polarizers. The theory of light propagation through a birefringent material is introduced to analyze the polarization of light within the cell, when its axes are oriented at $45^{\circ}$ to that of the polarizers. The period of modulation is derived, and the transmitted intensity is shown to oscillate as $\sin ^{2}(k V)$, where $k$ is some constant and $V$ the applied voltage. For a linear response, the modulating voltage has to be kept small, and the Pockels cell be biased at a voltage that yields an intensity halfway between the maximum and minimum values. Another optical arrangement which functions as an intensity modulator, is the Mach-Zehnder (MZ) interferometer. In this set-up, a ray passing through a Pockels cell is combined with a reference beam. A change in the voltage applied to the Pockels cell will result in a phase shift $\Delta \phi$ of light, so that one may obtain a full range of output from destructive $(\Delta \phi=\pi)$ to constructive $(\Delta \phi=0)$ interference.

The discussion on the MZ interferometer leads to the important topic of integrated optics (IO). The objective of IO technology is to perform various processing of the optical signals, such as focusing, combining, switching and polarization modulation on a single chip. Most IO elements are based on planar waveguides, formed e.g. by the diffusion of Ti in $\mathrm{LiNbO}_{3}$ to form a strip of raised refractive index. Waveguides can also be made of GaAs embedded in AlGaAs, which has a lower refractive index. The IO version of the MZ interferometer, consisting of a forked strip waveguide is compared to the conventional optical set-up. An obvious advantage of an IO device is its miniature size. Additionally, by using a longer strip waveguide, we can reduce the half-voltage to just a few volts, instead of hundreds of volts for a conventional Pockels cell. Yet another advantage is that many of the fabrication techniques used for integrated circuit technology can be readily adapted for the manufacture of IO devices. 
It was recently announced that Toshiba and Matsushita Electric Industrial Co. Ltd. will jointly build a polysilicon LC screen manufacturing plant in Singapore. This plant, touted as the world's largest, will begin full production from April 2003. In view of the expected demand for skilled workforce and expertise in this area, we have substantially expanded the course material on liquid crystals. Once again, a multi-disciplinary approach is taken, to give the student a broad overview of this vast and rapid developing topic. We begin with the basic chemistry of liquid crystals (LC), which is a special intermediate state of matter, having properties of both crystalline solids (e.g. orientational order) and liquids (e.g. lacking positional order). Structurally, LCs consist of long organic chains, made up of several functional groups. The dipolar and steric interactions between functional groups of neighboring chains cause LC molecules to align along a certain preferred axis ("director"). Since the interactions, which hold the LC molecules in place, are relatively weak, there is some degree of freedom about this director. Thus, LCs behave as a crystal whose symmetry axes may be easily modified by external means, and this accounts for its hybrid solid-liquid properties.

The orientational configuration of LCs comes in three main forms: smectic, cholesteric and nematic, of which the main focus is centered on the most useful type - the nematic LC. We introduce the mathematics used to characterize various properties of nematic LC, such as degree of orientational disorder, dielectric permittivity and refractive index. Due to its uniaxial structure, a nematic LC has a refractive index, which varies with the direction of light polarization, i.e. it exhibits birefringence.

The next section deals with the technologically important twisted nematic (TN) LC cell, in which the LC molecules are twisted along the length of the cell, by means of microgrooves on the surfaces of the cell. Some details on the material and fabrication of the TN cell are included, such as the sputtering of indium tin oxide on glass to act as a transparent electrodes sandwiching the LC material, and the use of polyimide film to etch the microgrooves. The physics behind the $\mathrm{TN}$ cell operation is also discussed, such as i) the equation of motion of the LC molecules, governed by viscous, intermolecular and electric forces, and ii) the electromagnetic interaction between the $E$ field of light and the dipole moment of the LC, which rotates the plane of polarization of light passing through. Based on these physics equations, measurable quantities such as threshold voltage and response time of TN cell are derived. More importantly, it is shown that by applying a bias of a few volts, the LC molecules can be aligned along the applied field and perpendicular to the plane of polarization of light. The causes the TN cell to change from an optically active to an inactive one. The TN cell can thus be converted into a voltage-controlled intensity modulator by placing it in between crossed polarizers. Due to the birefringent property of $\mathrm{TN}$ cell, it can also function as other electro-optic devices such as a wave retarder and a phase modulator.

The final section discusses the main present-day application of LCs, i.e. as liquid crystal displays (LCD). It begins with small-scale spatial light modulators, such as the seven-bar numeric display, to show how, by electrically addressing each segment, information can be displayed two-dimensionally. We then discuss the technology involved in large-scale LC displays that are currently being used in, e.g., notebook computers, and compare its performance with more established technologies, such as the cathode-ray tube. Next, the two modes of addressing are elaborated, i.e. i) passive and ii) active matrix. Both involve a crossbar address system, in which a TN cell (which forms a pixel element) is switched on by the coincident address of the column and row buslines intersecting at the electrode. The row busline is swept sequentially down the display (gate scan) while the state of the column (data) busline is determined by the desired output in the gate line being scanned. The main difference between the two modes is that in passive matrix display, there is no individual switch controlling each element. Therefore, the output at any one pixel is "diluted" by the signals sent to all other pixels along the same column in one full period of th gate scan. This reduces the difference in the time-averaged pixel voltage for the ON and OFF state, with the result that passive matrix displays suffer from a low contrast ratio. For active matrix display, each cell has its own electronic switch. The state of each cell is set when both data and gate buslines are addressed. The cell then retains this state until the next gate refresh, unaffected by data busline signals addressed to other rows, thus yielding a high degree of contrast. A description is given of the structure of the thin-film transistor (TFT), made of either amorphous or poly-Si, which forms the switch at each cell. Finally, to give a foretaste of the rapid developments in the field, we survey several novel types of LCDs and their respective strengths - supertwisted nematic LCD (brighter contrast and wider viewing angle), ferroelectric LCD (non-volatile memory, high response rate) and polymer-dispersed LCD (brightness and ease of fabrication). 


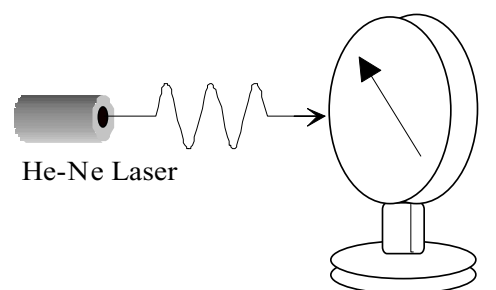

Variable

Polarizer at $45^{\circ}$

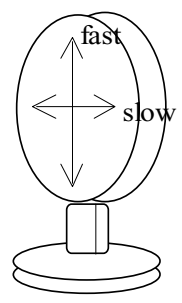

Variable LC retarder

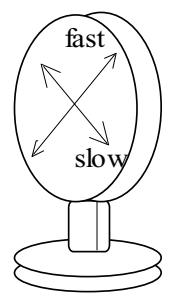

Quarter-wave plate with fast axis tilted $45^{\circ}$ to retarder's axes.

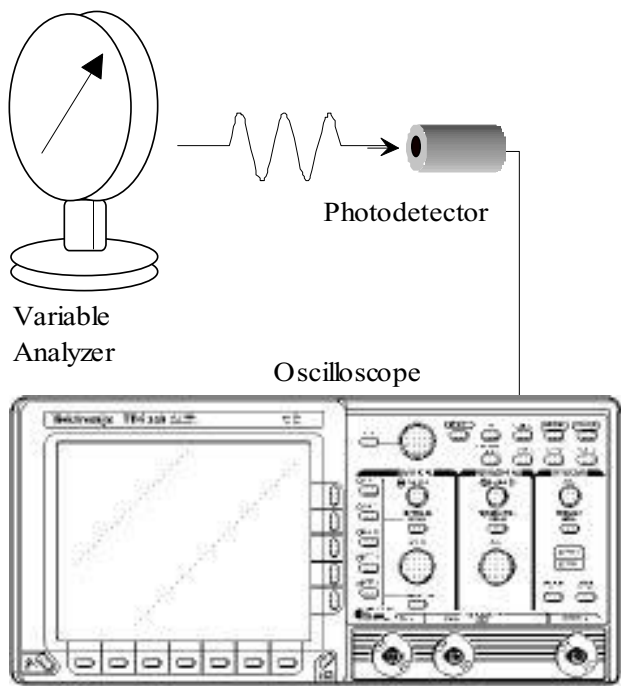

Figure 6. Optical set-up of LC experiment.

As a complement to the theory covered in the lectures, an experiment on the electro-optic and time response of an LC modulator has been designed, and will be introduced in the coming semester. The experiment is divided into two sections: i) investigation into the effect of the applied voltage $V$ on the phase retardation $\delta$ of light passing through the modulator, and ii) the determination of the rise $\tau_{\text {on }}$ and decay $\tau_{\text {off }}$ time constants of the LC molecules in the modulator. In part (i), $\delta$ is determined following two different methods. The first involves measuring the intensity of light passing through the set-up shown in Fig. 6 (but wihout the $\lambda / 4$ plate). The variation of light intensity versus applied voltage is shown in Fig. 7(a). In the second method, a $\lambda / 4$ plate is inserted in front of the analyzer, and $\delta$ is obtained from the resulting rotation of the plane of polarization. In part (ii) which measures the time response, a software package and a controller device are used to apply an a.c. voltage above the threshold to the LC cell [Fig. 7(b)]. Once the voltage is switched on, the rise time $\tau_{\text {on }}$ is determined by the time taken for the brightness to reach $90 \%$ of its maximum value, while the time for the brightness to decay to $10 \%$ after the voltage has been switched off gives the the decay time $\tau_{\text {off }}$. Finally, a waveform with an initial spike [Fig. 7(c)] is applied, in order to corroborate the theoretical prediction that this will lead to a faster response. ${ }^{3}$

\section{Conclusion}

An outline has been presented of a final-year undergraduate course conducted at NUS, Singapore. A multi-disciplinary approach encompassing solid state and device physics, optics, materials, and electronics, is adopted to give the student an overall view of this broad and expanding topic. The course material is constantly refreshed to incorporate the latest research and development in and out of NUS, and to meet the current needs of Singapore's fast-developing photonics industry. Experiments on lasers, LEDs and LC modulator provide a practical experience to complement the lectures.

\section{Acknowledgments}

M. B. A. Jalil acknowledges the financial support of the National University of Singapore (RP No. R263-000-029-112 and R263-000-029-303). 

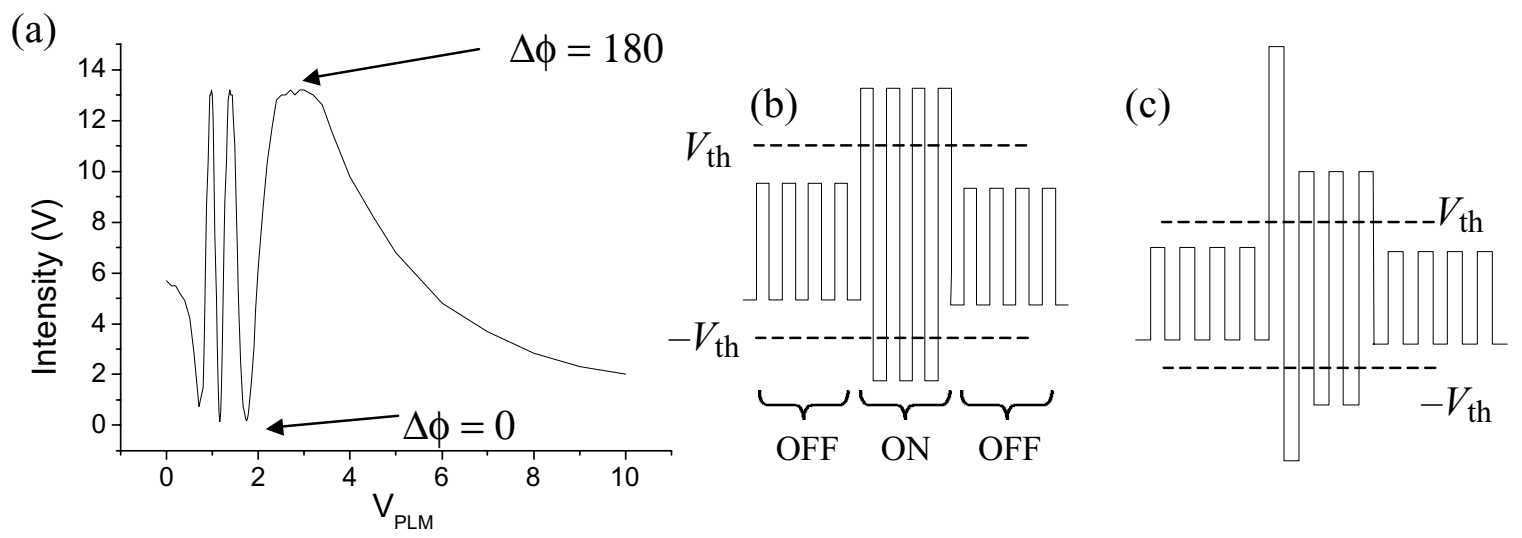

Fig. 7 (a). Transmitted intensity versus voltage $V_{\text {PLM }}$ applied to the LC modulator. (b) A.C. voltage signals applied on LC modulator to probe its time response. (c) Signal similar to (b) but with an initial spike.

\section{References}

${ }^{1}$ Chua SJ and Chong TC, "Current dependence of pulsation in semiconductor lasers due to photon induced modulation of optical loss”, IEEE J. Quant. Electron. Vol. QE-20, no. 11, pp. 1243-1246, 1984.

${ }^{2} \mathrm{Gu}$ X, Karunasiri G, Chen G, Sridhar U, Xu B, "Determination of thermal parameters of microbolometers using a single electrical measurement”, Appl. Phys. Letts. Vol. 72, pp. 1881-1883, 1998.

${ }^{3}$ Meadowlark Optics Manual, published by Meadowlark Optics Inc. p. 36, 1999. 